Check for updates

Cite this: RSC Adv., 2019, 9, 9887

Received 21st December 2018 Accepted 24th March 2019

DOI: $10.1039 / c 8 r a 10455 g$

rsc.li/rsc-advances

\section{Stereoselective analysis of the chiral fungicide penflufen in wheat plants, spinach, and Chinese cabbage $\uparrow$}

\author{
MingNa Sun, ${ }^{\mathrm{ab}}$ Zhou Tong, $^{\mathrm{ab}}$ Xu Dong, ${ }^{\mathrm{ab}}$ Yue Chu, $^{\mathrm{ab}}$ Mei Wang, ${ }^{\mathrm{ab}}$ TongChun Gao*ab $^{\star \mathrm{a}}$ \\ and JinSheng Duan (D) *ab
}

Penflufen is a highly efficient, broad-spectrum succinate dehydrogenase inhibitor. Owing to the increasing pesticide resistance in recent years, the use of a new fungicide, penflufen, has become increasingly widespread. However, residues that remain in the environment after the use of penflufen have an impact on human health. It is worth noting that penflufen is a chiral pesticide. The differences of residue behaviors between two enantiomers in living organisms need to be systematically studied. In this paper, reversed-phase liquid chromatography-mass spectrometry (LC-MS) was used to separate the enantiomers of penflufen, and the absolute configuration of the enantiomer was analyzed. The LC-MS/ MS methods for the analysis of penflufen enantiomers on wheat plants, spinach, and Chinese cabbage were established. The results of the recovery experiments showed that the average recovery of the two enantiomers was $78.5-99.8 \%$ and RSD was $0.4-7.3 \%$, suggesting that the accuracy and precision of the method meet the requirements of pesticide residue analysis. The results of stereoselective degradation of penflufen in the three matrices showed that there was little difference in the degradation of the two enantiomers in wheat and cabbage, while $R-(+)$-penflufen was degraded preferentially in spinach. This study provides data supporting the scientific use and safety evaluation of penflufen.

\section{Introduction}

A chiral pesticide is a pesticide that has at least one chiral central atom in its chemical structure. In non-chiral environments, chiral pesticides have the same physical and chemical properties. However, due to the existence of chiral characteristics in the ecological environment, different chiral pesticide enantiomers may have larger differences in environmental behavior, such as degradation, ${ }^{1-3}$ metabolism $^{4,5}$ and transformation. $^{6,7}$ Furthermore, the traditional pesticide risk assessment method cannot accurately assess the risk of chiral pesticides, which raises concern about safety hazards to humans and the environment.

Penflufen is an amide fungicide developed by Bayer AG. The chemical structure of penflufen was shown in Fig. 1, and the physical and chemical properties were listed in Table S1. $\dagger$ This fungicide is a succinate dehydrogenase inhibitor, which acts mainly on the respiratory chain of pathogens and affects their metabolism by blocking the respiratory chain. With the

${ }^{a}$ Institute of Plant Protection and Agro-Product Safety, Anhui Academy of Agricultural Sciences, Hefei 230031, China.E-mail: djsahzbs@163.com; gtczbs@sina.com ${ }^{b}$ Key Laboratory of Agro-Product Safety Risk Evaluation (Hefei), Ministry of Agriculture, Hefei 230031, China

$\dagger$ Electronic supplementary information (ESI) available. See DOI: $10.1039 / \mathrm{c} 8 \mathrm{ra10455g}$ widespread use of this type of fungicide on crops, its residual pollution is also becoming more and more serious. There are few reports on the quantitative analysis of penflufen in the environment. Tian et al. used UHPLC-MS/MS to establish a residue analysis method for penflufen and its metabolites in vegetables and cereals. ${ }^{8}$ However, the analytical methods of the

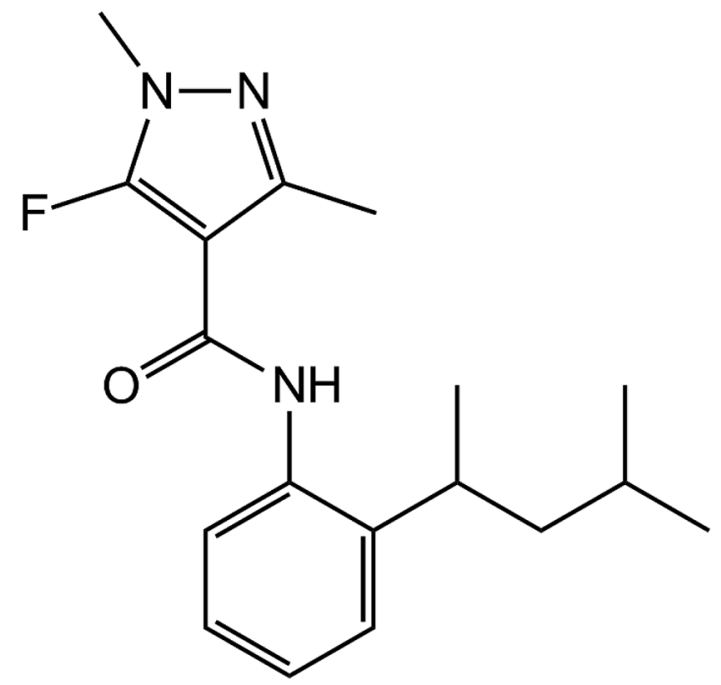

Fig. 1 The chemical structure of penflufen. 
chiral enantiomers of penflufen in the environment and the differences in the residual behavior of the enantiomers in the environment have not been studied. In this study, the chiral separation of penflufen was carried out by reversed-phase liquid chromatography coupled with a chiral column. The separation mechanism is considered that the polarity and $\pi-\pi$ effect in chiral columns were major factors in chiral separation. The absolute configuration of the enantiomers was determined by circular dichroism (ECD). Based on the studies and results, we used LC-MS/MS to establish a residue analysis method for the enantiomers of penflufen in wheat, spinach, and Chinese cabbage and studied the stereoselective degradation of two enantiomers in crops. The results of this study provide a theoretical basis for the rational use of penflufen and its safety assessment in the environment.

\section{Materials and methods}

\subsection{Standards and reagents}

The penflufen racemate standard $(98.72 \%$ of purity) was purchased from Dr Ehrenstorfer (Augsburg, Germany), and the optically pure enantiomer of penflufen (more than 95\% of purity) was prepared by Chiralway Biotech (Shanghai, China). The target compound which weighed and dissolved using methanol, was made into a standard solution with a concentration of $1000 \mathrm{mg} \mathrm{L}^{-1}$. The standard solution was gradually diluted with methanol and blank matrix solution, and stored at $-20{ }^{\circ} \mathrm{C}$.

Methanol, acetonitrile (HPLC grade, Tedia, USA), ammonium acetate and formic acid (HPLC grade) were obtained from Aladdin Bio-Chem Technology (Shanghai, China). N-Propylethylenediamine (PSA), and graphitized carbon black (GCB) were purchased from Agela Technologies (Tianjin, China). Anhydrous magnesium sulfate and sodium chloride (analytical grade) were all purchased from the National Pharmaceutical and Reagent (China).

\subsection{Instrument conditions}

LCMS-8030 high performance liquid chromatography-tandem mass spectrometer was purchased from Shimadzu (Japan), and a Lux $5 \mathrm{u}$ Cellulose- $2(150 \mathrm{~mm} \times 4.6 \mathrm{~mm}, 5 \mu \mathrm{m})$ chiral column with an amylose-tris[3,5-dimethylphenylcarbamate]coated chiral stationary phase was purchased from Feiluomen
Scientific Instrument (Guangzhou, China). The chromatographic separation conditions were as follows: mobile phase A was $0.1 \%$ formic acid aqueous solution; mobile phase B was

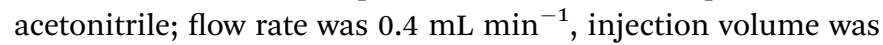
$10 \mu \mathrm{L}$; column temperature was $35{ }^{\circ} \mathrm{C}$; gradient elution procedure is shown in Table S2; $\uparrow$ mass spectrometry was performed using positive ion ionization (ESI+) of electrospray ion source; capillary voltage was $4.5 \mathrm{kV}$; desolvation tube temperature was $250{ }^{\circ} \mathrm{C}$; heating module temperature was $450{ }^{\circ} \mathrm{C}$; both atomizing gas and drying gas were high purity nitrogen; flow rate were 3.0 and $20 \mathrm{~L} \mathrm{~h}^{-1}$, respectively; the collision gas is high purity argon. The mass spectrometric conditions of the target compound in MRM mode are shown in Table S3. $\dagger$

\subsection{Determination of rotation and absolute configuration for penflufen enantiomers}

The specific rotation of the penflufen enantiomer was detected by a WZZ-2S automatic polarimeter. The penflufen enantiomer was dissolved in methanol at a concentration of $5000 \mathrm{mg} \mathrm{L}^{-1}$. Methanol was used as control (CK). After the polarimeter was adjusted, the monomer solution samples were measured three times. The test results were temperature-corrected and the mean value of three measurements was calculated.

Penflufen solution was diluted to $100 \mathrm{mg} \mathrm{L}^{-1}$ with methanol, and then the electronic circular dichroism (ECD) was measured. The circular dichroism polarimeter was zeroed, and the solution of penflufen enantiomer was scanned at $20{ }^{\circ} \mathrm{C}$ using a circular dichroism polarimeter in the range of $200-400 \mathrm{~nm}$ to draw a theoretical ECD spectrum. The density functional theory (DFT) B3LYP method was used to calculate the circular dichroism spectrum, and the Gaussian 09 calculation method in time-dependent DFT (TDDFT) [B3LYP/6-31+G*] was used to calculate the ECD spectrum.

\subsection{Field trials}

The field trial was conducted in Hefei, Anhui Province, China. The wheat, spinach, and cabbage fields that had not previously been treated with penflufen were selected as experimental plots. The experiment was set up with three replicates, and a blank control plot was set up. Each plot was $30 \mathrm{~m}^{2}$, and $22 \%$ of the penflufen suspension was sprayed in the field at a dose of $300 \mathrm{~g}$ a.i. ha ${ }^{-1}$. All the samples were collected from the aerial parts of the plant. In each treatment, $2 \mathrm{~kg}$ weight of the wheat plants,

Table 1 Solvent standard curve and matrix standard curve data of penflufen enantiomers

\begin{tabular}{|c|c|c|c|c|c|}
\hline \multirow[t]{3}{*}{$S$-(-)-Penflufen } & Acetonitrile & 0.9986 & - & 0.5 & 1.7 \\
\hline & Wheat plant & 0.9998 & 21.2 & 6.0 & 20 \\
\hline & Chinese cabbage & 0.9983 & 28.1 & 6.0 & 20 \\
\hline \multirow[t]{2}{*}{$R-(+)$-Penflufen } & Acetonitrile & 0.9996 & - & 0.5 & 1.7 \\
\hline & Wheat plant & 0.9992 & 33.9 & 6.0 & 20 \\
\hline
\end{tabular}


Table 2 Accuracy and precision of penflufen enantiomers in three matrices $(n=5)$

\begin{tabular}{|c|c|c|c|c|c|}
\hline \multirow[b]{2}{*}{ Matrix } & \multirow[b]{2}{*}{$\begin{array}{l}\text { Spiked concentration } \\
\left(\mathrm{mg} \mathrm{kg}^{-1}\right)\end{array}$} & \multicolumn{2}{|l|}{$S$-(-)-Penflufen } & \multicolumn{2}{|l|}{$R$-(+)-Penflufen } \\
\hline & & Average recovery (\%) & RSD (\%) & $\begin{array}{l}\text { Average recovery } \\
(\%)\end{array}$ & $\operatorname{RSD}(\%)$ \\
\hline \multirow[t]{3}{*}{ Wheat plant } & 2.0 & 97.4 & 1.7 & 96.8 & 0.4 \\
\hline & 0.2 & 89.5 & 6.5 & 91.6 & 3.5 \\
\hline & 0.02 & 86.1 & 1.9 & 78.5 & 1.6 \\
\hline & 0.02 & 84.2 & 4.2 & 79.2 & 2.2 \\
\hline \multirow[t]{3}{*}{ Chinese cabbage } & 2.0 & 93.9 & 2.8 & 98.8 & 2.4 \\
\hline & 0.2 & 87.4 & 2.8 & 89.1 & 2.0 \\
\hline & 0.02 & 87.0 & 6.3 & 80.2 & 3.7 \\
\hline
\end{tabular}

Table 3 Half-life of penflufen enantiomers

\begin{tabular}{llll}
\hline Matrix & Enantiomer & Half-life (days) & Kinetic equation \\
\hline Wheat plant & $S$-(-)-Penflufen & 4.74 & \multicolumn{1}{c}{$R^{2}$} \\
& $R$-(+)-Penflufen & 4.66 & $1.4508 \times 10^{-0.146 x}$ \\
Spinach & $S$-(-)-Penflufen & 3.57 & 0.9326 \\
& $R$-(+)-Penflufen & 3.15 & $y=35.17 \times 10^{-0.194 x}$ \\
Chinese cabbage & $S$-(-)-Penflufen & 3.92 & $y=30.364 \times 10^{-0.22 x}$ \\
& $R$-(+)-Penflufen & 3.77 & $y=32.43 \times 10^{-0.177 x}$ \\
& & & $y=28.919 \times 10^{-0.184 x}$
\end{tabular}
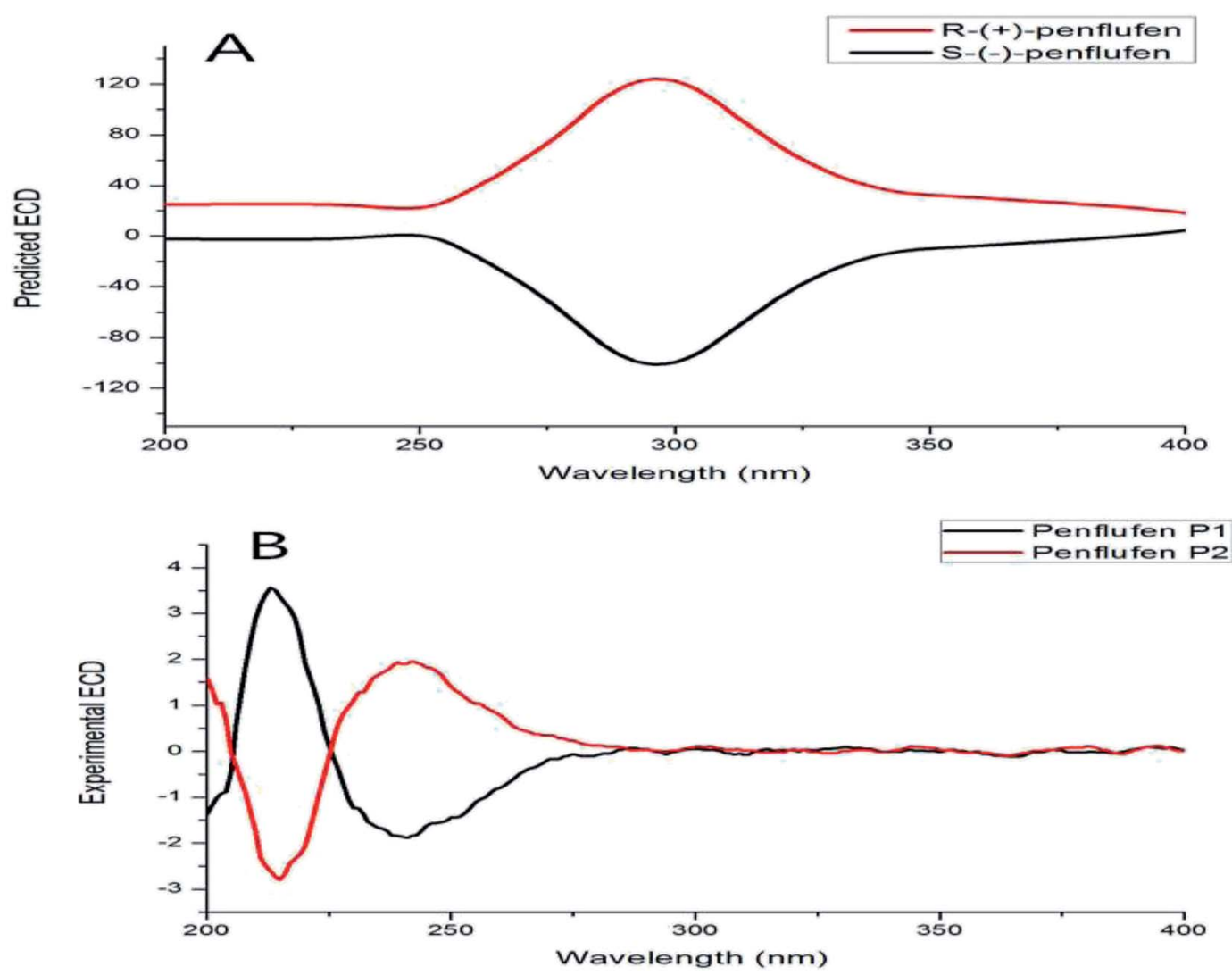

Fig. 2 Predicted and experimentally measured ECD spectra of penflufen enantiomers: (A) predicted ECD spectra and (B) experimentally measured ECD spectra. 
spinach, and cabbage samples were collected at $2 \mathrm{~h}, 1 \mathrm{~d}, 3 \mathrm{~d}, 5 \mathrm{~d}$, $7 \mathrm{~d}, 10 \mathrm{~d}, 14 \mathrm{~d}, 21 \mathrm{~d}$, and $30 \mathrm{~d}$ after treatment, and stored at $-20{ }^{\circ} \mathrm{C}$ for testing.

\subsection{Sample pretreatment}

An accurately weighed sample (5 g) was put into a $50 \mathrm{~mL}$ centrifuge tube. $10 \mathrm{~mL}$ of water and $20 \mathrm{~mL}$ of acetonitrile were added into the tube. The sample was extracted for $30 \mathrm{~min}$ with shaking. Then, $3 \mathrm{~g}$ of anhydrous magnesium sulfate and $1 \mathrm{~g}$ of sodium chloride were added into the tube. The tube was shaken vigorously for $2 \mathrm{~min}$ and centrifuged at $3000 \mathrm{rpm}$ for $5 \mathrm{~min}$. Five microliters of the upper organic phase was pipetted into a $10 \mathrm{~mL}$ centrifuge tube (containing $250 \mathrm{mg}$ of PSA, $750 \mathrm{mg}$ of anhydrous magnesium sulfate, and $20 \mathrm{mg}$ of GCB) and vortexed for $1 \mathrm{~min}$. The mixture was centrifuged at $5000 \mathrm{rpm}$ for $3 \mathrm{~min}$. The supernatant was passed through a $0.22 \mu \mathrm{m}$ micropore filter to the injection vial and tested by LC-MS/MS.

\subsection{Data analysis}

Matrix effects (MEs) were calculated between slopes of the matrix calibration curve and the solvent calibration curve for every determination.

$\operatorname{ME}(\%)=\left(\left(\frac{\text { slope of calibration curve in matrix }}{\text { slope of calibration curve in solvent }}\right)-1\right) \times 100$

The enantiomeric fraction (EF) value was used for characterizing the stereoselectivity of penflufen degradation in the environment

$$
\begin{aligned}
& \mathrm{EF}= \\
& \frac{\text { area of }(-) \text {-penflufen peak }}{\text { area of }(-) \text {-penflufen peak }+ \text { area of }(+) \text {-penflufen peak }}
\end{aligned}
$$

The $\mathrm{EF}$ value ranges from 0 to 1 , and $\mathrm{EF}=0.5$ indicates that the two enantiomers have the same rate of degradation and no enantioselectivity. When $\mathrm{EF}>0.5$, it indicates that the (+)-enantiomer is preferentially degraded; when $\mathrm{EF}<0.5$, it indicates that the (-)-enantiomer is preferentially degraded.

The degradation rate constants of penflufen enantiomers in plants and soil were calculated by the first-order kinetic equations $(k)$.

The relationship between residual concentration and time is as follows:

$$
C=C_{0} \mathrm{e}^{-k t},
$$

where $C_{0}$ is the initial concentration of penflufen enantiomer in the sample after administration $\left(\mathrm{mg} \mathrm{kg}^{-1}\right), C$ is the concentration of penflufen enantiomer at the time $t$ after administration $\left(\mathrm{mg} \mathrm{kg}^{-1}\right), k$ is the degradation rate constant, and $t$ is the time after administration. The degraded half-life time $\left(T_{1 / 2}\right)$ of penflufen enantiomer can be estimated by the obtained $k$, and the calculation formula is as follows: $T_{1 / 2}=\ln 2 / k=0.693 / k$.

\section{Results and discussion}

\subsection{Elution order and absolute configuration}

The specific rotation of the first eluted enantiomer of penflufen in liquid chromatography was $[\alpha]_{\mathrm{D}}^{25}=-1.72$; the specific rotation of the second eluted chromatographic peak was $[\alpha]_{\mathrm{D}}^{25}=$ +1.63. Comparison between the theoretical ECD spectrum and the calculated ECD spectrum in Fig. 1 showed that the theoretical ECD spectrum at $250-300 \mathrm{~nm}$ is similar to the calculated ECD at 220-270 $\mathrm{nm}$. Combined with the peak optical rotation of penflufen eluted, it was estimated that the first enantiomer of penflufen is $S$-(-)-penflufen and the second one is $R$-(+)-penflufen in Fig. 1.

\subsection{Linearity, limit of quantification, and matrix effect}

The enantiomeric linearity of penflufen is in the range of $0.02-$ $10 \mathrm{mg} \mathrm{L}{ }^{-1}$. The linear equations of the enantiomers of penflufen in solvent, wheat plant samples, spinach samples, and Chinese cabbage samples are shown in Table 1. The linear correlation coefficient is between 0.9983 and 0.9999 , which

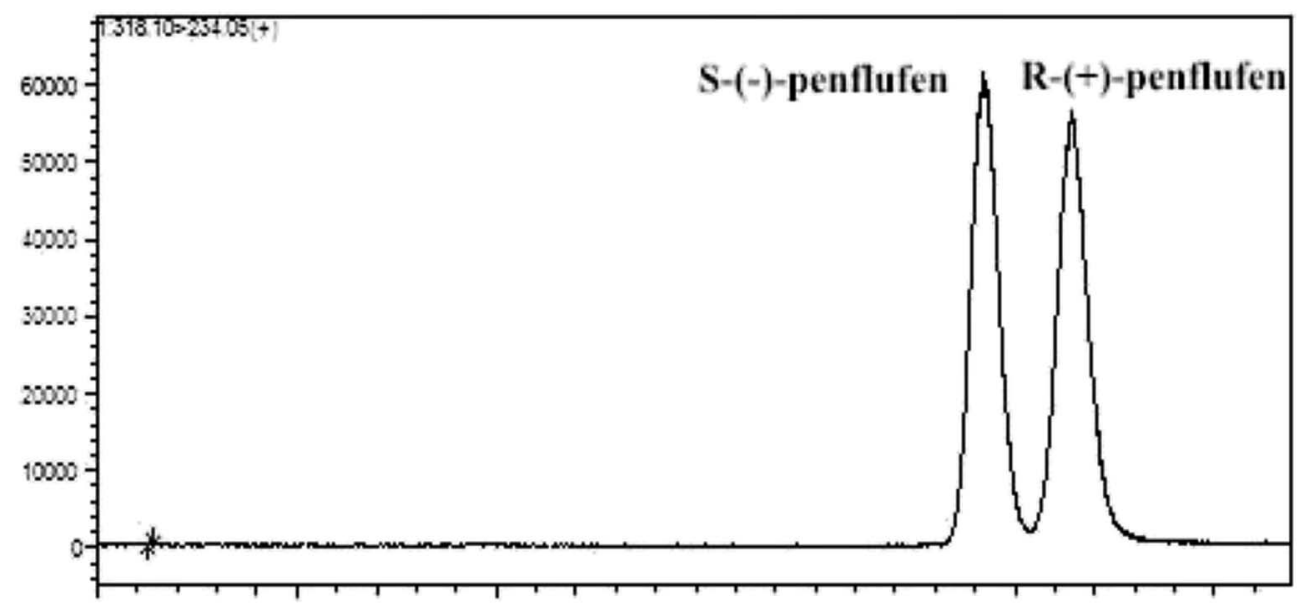

Fig. 3 LC-MS/MS chromatogram of two penflufen enantiomeric standard samples. 

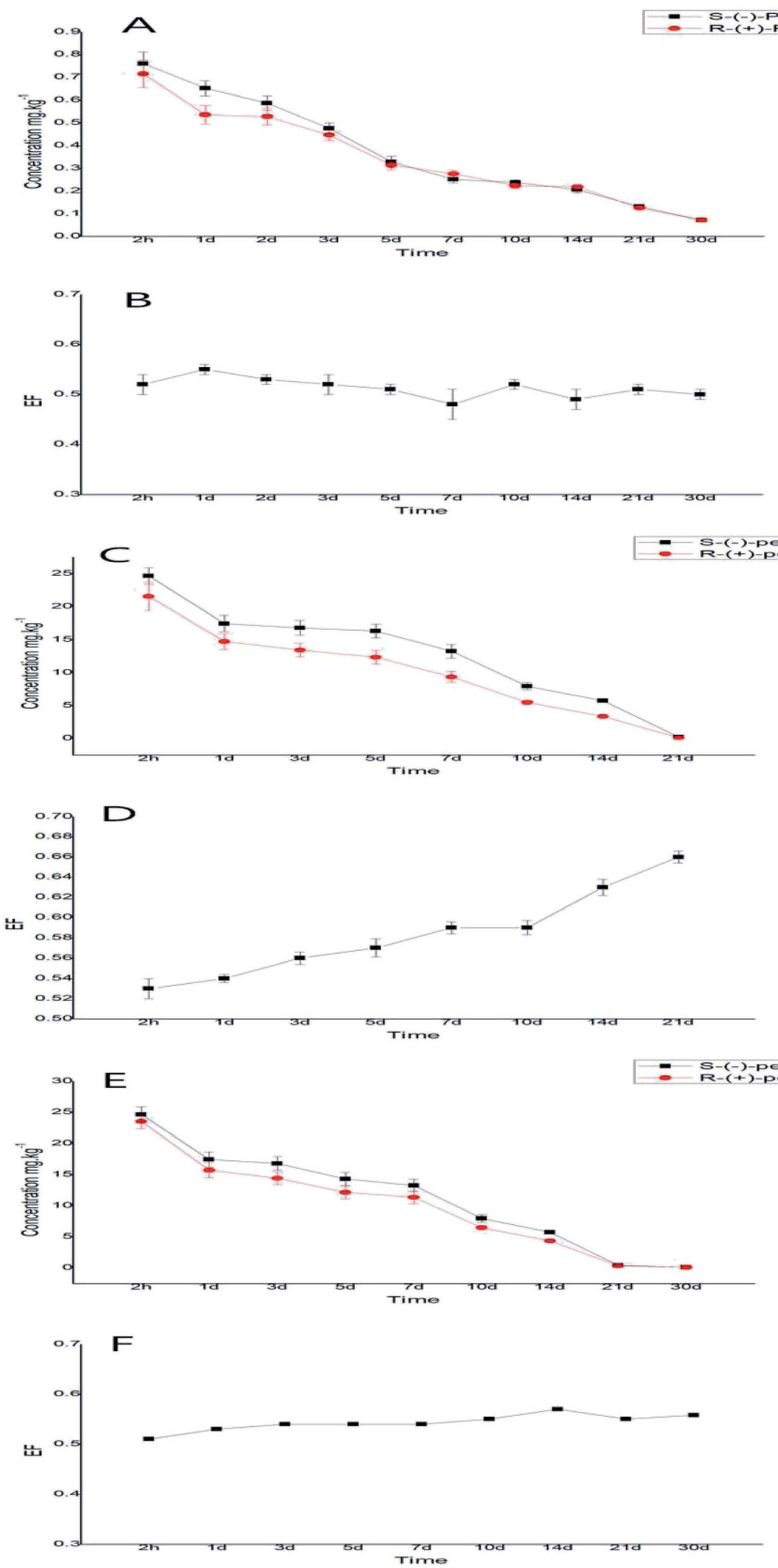

Fig. 4 Stereoselective degradation of penflufen on wheat plant, spinach, and Chinese cabbage (wheat plants: A and B; spinach: C and D; Chinese cabbage: $\mathrm{E}$ and F). 
means a good correlation. Therefore, the external standard method was used to quantitatively analyze the two enantiomers in the samples here. In previous study, the LOQ of parent penflufen was ranged from $16 \mu \mathrm{g} \mathrm{kg}^{-1}$ to $42 \mu \mathrm{g} \mathrm{kg}{ }^{-1}$ in vegetables and cereals. Meanwhile, the limit of quantification of the penflufen enantiomers in different samples was above $20 \mu \mathrm{g}$ $\mathrm{kg}^{-1}$, and the sensitivity of the instrument meet the requirements of trace analysis. At the same time, the enantiomers of penflufen had different levels of matrix effects in the samples; as a result, a standard solution was prepared using a blank matrix to reduce the interference of the matrix effect during quantification.

\subsection{Method validation}

Three concentrations of penflufen $\left(0.02,0.2\right.$ and $2.0 \mathrm{mg} \mathrm{kg}^{-1}$ ) were added to wheat plant, spinach, and Chinese cabbage samples with five treatments. The average recovery of penflufen enantiomers in the three matrixes was 78.5-99.8\%, and the RSD was $0.4-7.3 \%$ (shown in Table 2). The results showed that the method has a good accuracy and precision, which can meet the needs of stereoselectivity research.

\subsection{Stereoselective behavior}

After the wheat plants were sprayed with penflufen, the half-life of penflufen enantiomer degradation in the wheat plant samples was determined, as shown in Table 3. It can be found that the half-lives of $S$-(-)-penflufen and $R$-(+)-penflufen in the wheat plants were $4.74 \mathrm{~d}$ and $4.66 \mathrm{~d}$, respectively. The degradation rate of $R-(+)$-penflufen is basically consistent with that of $S$-(-)-penflufen. The EF value of penflufen enantiomers at each sampling time point was calculated as shown in Fig. 2. It can be seen that the EF value of penflufen enantiomers was 0.48-0.54, indicating that there is no selective degradation of penflufen enantiomers on wheat plants.

The degradation rate of penflufen enantiomers in spinach samples fit with the first-order kinetic equation. Penflufen could not be detected on the 30th day. The degradation half-life of penflufen enantiomers in spinach samples is shown in Table 3 , and the half-lives of $S$-(-)-penflufen and $R-(+)$-penflufen in spinach samples were $3.57 \mathrm{~d}$ and $3.15 \mathrm{~d}$, respectively. The EF value at each sampling time point was calculated in this study (Fig. 4). The results in Fig. 3 showed that the EF of penflufen enantiomers increased from 0.5 to 0.63 with the prolongation of the time after application. An increase in the interval EF value was observed, indicating that the enantiomer of penflufen had obvious selective degradation on spinach plants over time. $S$ (-)-penflufen has a relatively slower degradation rate and more accumulation in spinach.

The results of selective degradation analysis of penflufen on Chinese cabbage showed that the half-lives of the enantiomers of penflufen in Chinese cabbage were $3.92 \mathrm{~d}$ and $3.79 \mathrm{~d}$, respectively. The degradation rate of $S$-(+)-penflufen is basically consistent with that of $R$-(-)-penflufen. The EF value of penflufen enantiomers was $0.5-0.57$ with the prolongation of the time after administration, indicating that the selective degradation of penflufen enantiomers on Chinese cabbage was not obvious. The mechanism of stereoselective degradation was reported in some previous studies, the enzymatic systems has been considered that is the major factor in plants. ${ }^{9-11}$

\section{Conclusion}

The chiral separation method of the new chiral fungicide, penflufen, by LC-MS/MS was studied in this study, and the analytical methods of penflufen enantiomers in wheat plants and two vegetables are established here. Studies on the selective degradation in a farmland environment showed that the enantiomer of penflufen was selectively degraded on spinach. Our results provide data supporting the rational use of penflufen in the environment.

\section{Conflicts of interest}

The authors declare no competing financial interest.

\section{Acknowledgements}

This work was supported by the National Key Research and Development Program of China (2016YFD0200207).

\section{References}

1 I. J. Buerge, T. Poiger, M. D. Müller and H. R. Buser, Environ. Sci. Technol., 2003, 37, 2668-2674.

2 H. R. Buser, M. D. Müller, T. Poiger and M. E. Balmer, Environ. Sci. Technol., 2002, 36, 221-226.

3 Z. Y. Li, Y. C. Zhang, Q. L. Li, W. X. Wang and J. Y. Li, Environ. Sci. Technol., 2011, 45, 2797-2803.

4 T. A. Müller and H. P. Kohler, Appl. Microbiol. Biotechnol., 2004, 64, 300.

5 S. M. Sanins, W. J. Adams, D. G. Kaiser, G. W. Halstead, J. Hosley, H. Barnes and T. A. Baillie, Drug Metab. Dispos., 1991, 19, 405.

6 J. L. Jarman, W. J. Jones, L. A. Howell and A. W. Garrison, J. Agric. Food Chem., 2005, 53, 6175-6182.

7 T. Lv, P. N. Carvalho, M. E. Casas, U. E. Bollmann, C. A. Arias, H. Brix and K. Bester, Environ. Pollut., 2017, 229, 362.

8 F. Tian, X. Liu, Y. Wu, J. Xu, F. Dong, X. Wu and Y. Zheng, Food Chem., 2016, 213, 410-416.

9 D. Fengshou, L. Jing, C. Bezhan, C. Yongpu, X. Jun, L. Xingang, L. Yuanbo, C. Xiu, B. Carlo and T. Daniele, Environ. Sci. Technol., 2013, 47, 3386-3394.

10 D. Fengshou, C. Li, L. Xingang, X. Jun, L. Jing, L. Yuanbo, K. Zhiqiang, J. Qiu and Z. Yongquan, J. Agric. Food Chem., 2012, 60, 1929-1936.

11 B. Gao, Z. Zhang, L. Li, A. E. Kaziem, Z. He, Q. Yang, P. Qing, Q. Zhang and M. Wang, Sci. Total Environ., 2019, 648, 703710. 